\title{
UHMWPE for arthroplasty: past or future?
}

\author{
Elena Maria Brach del Prever • Alessandro Bistolfi • \\ Pierangiola Bracco $\cdot$ Luigi Costa
}

Received: 2 September 2008/ Accepted: 14 November 2008/Published online: 24 December 2008

(C) Springer-Verlag 2008

\begin{abstract}
Wear debris related osteolysis is recognised as being the main cause of failure in joint replacements based on UHMWPE inserts. However, many solutions and "new" polyethylenes have been suggested in order to address this issue. This review discusses "historical" issues associated with UHMWPE, such as oxidation, sterilization method and storage, as well as "new" topics, such as crosslinking and stabilization. The final aim is to aid orthopaedic surgeons in their selection of polyethylene inserts and in the information given to the patients. The main problem for the polymer is degradative oxidation, which is caused by the combination of the irradiation used for sterilization and oxygen, and which leads to a decrease in wear resistance and mechanical properties. Irradiation and packaging in the absence of oxygen can only reduce the oxidation, while sterilization with gas (EtO or gas plasma) is the only method that effectively eliminates it. Manufacturing processes are of great relevance to the clinical duration and must be considered by surgeons. Crosslinked polyethylene has been developed for joint inserts due to its superior wear resistance compared to conventional UHMWPE; to prevent the oxidation, crosslinked polyethylene requires post-irradiation thermal
\end{abstract}

\section{E. M. Brach del Prever ( $\square)$}

Dipartimento di Traumatologia, Ortopedia e Medicina del Lavoro dell'Università degli Studi di Torino, Centro Traumatologico Ortopedico, Via Zuretti 29, 10126 Torino, Italy e-mail: elena.brach@unito.it

\author{
A. Bistolfi \\ AO CTO/M. Adelaide, Via Zuretti 29, 10126 Torino, Italy \\ P. Bracco $\cdot$ L. Costa \\ Dipartimento di Chimica, Inorganica e Chimica Fisica \\ dell'Università di Torino and NIS Centre of Excellence, \\ Via P. Giuria 7, 10125 Torino, Italy
}

treatment, which reduces its mechanical properties and which depends on the producer. Several good clinical results from the use of crosslinked acetabular cups have reported at mid-term, while early results for knee replacements are also encouraging. Recently, the use of the antioxidant vitamin E (alpha-tocopherol) has been introduced for joint prostheses in order to prevent the oxidation of both crosslinked and noncrosslinked UHMWPE.

Keywords UHMWPE - Crosslinked · Vitamin E · Oxidation

\section{Introduction}

Four decades after its introduction for joint arthroplasties, UHMWPE still represents the gold standard as an articulating counterface for arthroplasties, since it combines superior wear resistance along with high fracture toughness and biocompatibility compared to other polymers.

It has been demonstrated that the main factor responsible for the failure of UHMWPE in joint replacements is oxidative degradation, which decreases its mechanical properties [1-3]. Decreased abrasive wear resistance, due to oxidation, leads to the formation of wear debris and consequently to osteolysis, which has been recognized as being the main cause of failure in orthopaedic implants [14].

The mechanical properties are of great relevance when the UHMWPE inserts are subjected to high contact stresses that can exceed the yield stress of the UHMWPE, leading to permanent deformation and to the catastrophic rupture and failure of the implant [5].

Oxidation is strictly correlated with the sterilization method: UHMWPE components sterilized with ethylene 
oxide (EtO) do not oxidize, while those sterilized using high-energy radiation in air $(\gamma$ radiation or an electron beam with a dose of 25-40 kGy) are known to show high levels of oxidation [3, 6-9]. Immediate oxidation following the high-energy treatment results in chain scission in the UHMWPE, thus immediately decreasing its molecular mass and therefore its mechanical properties. Moreover, the oxidative degradation proceeds during storage and in vivo, thus further exacerbating the problem [8-10]. Several methods have been applied to reduce the impact of the oxidation on the duration of the implant: thicker inserts have been recommended, the designs of the implants have been improved, and the use of ionizing radiations in air for sterilization has been avoided.

In 1998, in the Safety Notice 9816-1998 (UHMWPE Components of Joint Replacement Implants), the British Medical Devices Agency established that UHMWPE components should not be used if they are over five years old and if they have passed their indicated expiry date. In 2005, the Italian Ministero della Salute (see DGFDM/III/ 7101/P/I.1.c.r. 8/3/2005) recommended that the use of implants sterilized in the presence of oxygen should be avoided.

Recently, radiation crosslinking of the UHMWPE has been applied as answer to the main problem, wear debris related osteolysis [11]. Alternative bearing materials, such as ceramics and metals, are associated with concerns about biocompatibility, duration, carcinogenicity, revision difficulties and costs, which explains why joint replacements using polyethylene inserts are still the ones mostly commonly used in orthopaedics.

This review discusses the "historical" issues associated with polyethylene, such as oxidation, sterilization method and storage, as well as "new" topics, such as crosslinking and stabilization. The final aim is to aid the orthopaedic surgeons in the selection of the implant and polyethylene insert, the information given to the patient, and when signing contracts.

\section{UHMWPE: the material and its properties}

Medical-grade polyethylene (UHMWPE with an average molecular mass of $>2,000,000$ a.m.u.) is a semicrystalline polymer that can be depicted as a set of ordered regions (crystalline lamellae) embedded in a disordered amorphous phase [12]. The degree of crystallinity is an important parameter: higher crystallinity gives a larger modulus of elasticity, superior yield strength, improved resistance to creep deformation and enhanced fatigue strength, all of which are desirable properties for joint components. The degree of crystallinity, within the range commonly used for medical-grade UHMWPE, does not substantially affect the wear resistance, which is related to the molecular mass [3]. The resistance to creep deformation of the UHMWPE is important to evaluations of the relative contribution of deformation or wear to the penetration of the femoral head into the insert. The fatigue strength is also very important, since it relates to the ability of UHMWPE to resist cyclic damage modes, which are very common in knee components and also in hip components, although prevalent in the rims of malpositioned cups.

Medical-grade UHMWPE orthopaedic implants are machined from stocks and sheets made from UHMWPE powders by compression moulding or ram extrusion and subsequent annealing [1]; the ASTM F 648-07 designation (standard specification for UHMWPE powder and fabricated form for surgical implants) defines the characteristics required for medical-grade orthopaedic UHMWPE: density excluded, there are no upper limits on any of the starting parameters, and the characteristics of the material are determined before processing and sterilization. It is clear that commercially available UHMWPE inserts can be very different from each other after processing, sterilization and packaging, which is very relevant to their clinical applications.

\section{Oxidative degradation}

When a polymeric material is exposed to a stronger energy than that of the chemical bonds, the consequence is bond scission and the formation of free radicals; this chain fragmentation modifies the mechanical properties of the polymer [13]. High-energy radiation ( $\gamma$-rays, X-rays and electron beams), heat and strong mechanical stress are all examples of energies that can break chemical bonds. Even if only a single $\mathrm{C}-\mathrm{C}$ bond of the polymer chain in UHMWPE is broken and two $\mathrm{CH}_{2}$ - radicals are formed, the molecular mass decreases; as a consequence, many of the chemical and physical properties of the polymer begin to worsen.

In orthopaedics, this issue is mainly associated with the $\gamma$ radiation and electron beams commonly used during sterilization, and the process is known as degradation. If oxygen is present when the degradation process occurs, it is called oxidative degradation (oxidation). Once the oxidation process (which is also a function of the temperature) has been initiated, it cannot be interrupted, and its rate increases continuously with a series of reactions that involve free radicals and oxygen. The extent of the oxidative process depends on the number of radicals formed during sterilization and on the amount of oxygen, which can be either atmospheric, present at the sterilization, or it can be oxygen that penetrated by diffusion into the polymer during processing and storage or while the joint is being 
used in vivo. Therefore, the oxidative degradation can continue during storage and in vivo implantation $[14,15]$.

\section{Sterilization and packaging issues}

Sterilization of UHMWPE components deserves a special mention since, as described above, it is known that this process can modify the mechanical and wear properties of UHMWPE $[1,6-9,16]$.

Obviously, finished UHMWPE orthopaedic components must undergo sterilization before clinical use. High-energy radiation represents the most common sterilization technique: the source of $\gamma$ radiation is the decay of an unstable ${ }^{60}$ Co nucleus, while electron beams are generated from the electrons emitted by a thermally excited tungsten filament, which are accelerated by electric fields. The dose absorbed by the material during sterilization depends on the geometry of the sample and its position in relation to the source. The electron beam is easier to control and requires a shorter period of treatment (seconds).

UHMWPE components are usually stored on the shelf for long durations prior to implantation (periods of six months or longer); in addition, UHMWPE inserts of total joint replacements have historically been packaged in air and thereafter sterilized by $\gamma$ radiation. It is well established that such irradiation, as well as electron beam irradiation, causes crosslinking, chain scission and long-term oxidative degradation of polyethylene, and that long-term post-irradiation aging can have detrimental effects on the morphology and mechanical properties of UHMWPE [1, 14, 16, 17].

Macroscopic evidence for the oxidative degradation caused by $\gamma$-sterilization in air can be seen on a UHMWPE section, where it looks like a white halo and is called the "crown effect" or white band; this is a zone where a critical molecular mass decrease has occurred, and which therefore has very low mechanical properties, resulting in the well-known effects of delamination and fracture that are typical of such components [18]. It was previously erroneously believed that oxidation was associated with fatigue damage mechanisms; however, it has since been established that there is a correlation between the rate of abrasive wear and the post-oxidative reduction in molecular weight $[2,3]$.

In response to these oxidation issues, some manufacturers now sterilize UHMWPE using non-radiation-based methods, such as ethylene oxide (EtO) or (more recently) gas plasma sterilization (GP); sterilization by steam is not feasible because the temperatures required-about $135^{\circ} \mathrm{C}$ - could result in modifications to the material.

EtO is used to sterilize UHMWPE components sealed in gas permeable packages. The treatment is continued for as long as needed for the gas to diffuse inside the containers; the packages are then left under vacuum for enough time to allow the complete elimination of EtO. Prosthetic UHMWPE sterilized with EtO does not undergo any variation in chemical and physical structure.

Gas plasma is a surface sterilization method based on the action of ionized gas (i.e., hydrogen peroxide or peracetic acid), which deactivates biological organisms. Commercially available GP sterilization methods are usually carried out at low temperatures (below $50^{\circ} \mathrm{C}$ ) and do not significantly affect the physical, chemical and mechanical properties of UHMWPE.

A detailed mechanism for the oxidation for orthopaedic implants has been described, and it has been demonstrated that oxidation can also occur under certain conditions in ethylene oxide sterilized UHMWPE, albeit to a much smaller extent than for $\gamma$-radiation-sterilized UHMWPE. However, this phenomenon has been related to the presence in the pristine resin of calcium stearate, which is no longer used in contemporary medical-grade UHMWPE [7-9].

As another response to long-term post-irradiation ageing and oxidation, some manufacturers have recently shifted to sterilization with high-energy radiation performed in vacuum or under inert gases (nitrogen or argon).

The material used for the envelope or packaging itselfwhich can classified into the following categories: (a) gaspermeable packaging; (b) polymer barrier packaging, and; (c) aluminium barrier packaging-is clearly important.

The gas-permeable packaging used is usually a PET (polyethylene terephthalate) blister with a Tyvek ${ }^{\circledR}$ cover, which allows the diffusion of gases (oxygen included); it is therefore indicated for EtO or gas plasma sterilization, but it does not prevent oxidative degradation when used for radiation sterilization.

Polymer barrier packaging is based on a series of multilayer plastic bags with gas-barrier properties, and therefore has a limited but measurable permeability to oxygen; it does not exclude the presence of oxygen during and after the radiation sterilization.

Aluminium barrier packaging is virtually impermeable to gases, and so only oxygen already dissolved in the UHMWPE prior to irradiation can be present.

A complete absence of sterilization-induced oxidation can only be guaranteed by gas sterilization, particularly because the extent to which in vivo oxidation rates affect the clinical performance of conventional UHMWPE packaged in low-oxygen environments and then sterilized using $\gamma$ radiation is still unclear.

\section{Wear and debris}

Abrasion wear is the process of removing parts of a material from the surface during reciprocal movement 
along another surface with greater hardness. In orthopaedic joint components, the UHMWPE is removed because the interactions of its chains are weak compared to those between the metal or ceramic atoms in femoral head and femoral knee components.

The particles of polyethylene removed induce aseptic loosening, through a mechanism involving the formation of reactive tissue and consequently osteolysis, which has been recognized as being the main cause of implant failure [4, 19]. The exact immune reaction that occurs in periprosthetic osteolysis of joint replacements is still unclear: it is known that several types of immune processes appear to be relevant. A foreign-body, granulomatous response to UHMWPE particles denotes a nonspecific chronic inflammatory reaction involving activated mononucleated macrophages and fibroblasts but few T lymphocytes [20].

The activation of macrophages has been related to the size, shape, volume and number of radiation-sterilized UHMWPE debris particles: those $0.3-10 \mu \mathrm{m}$ in size are phagocytable and are therefore the most biologically active [21, 22]. An influence of the chemical composition of the UHMWPE particles has recently been suggested: the reactivity might be related to the composition of the surfaces of the particles themselves (superficial reactivity), and in particular to the level of oxidation of the UHMWPE itself $[23,24]$. Oxidized particles from $\gamma$-irradiated UHMWPE inserts would be more effective at activating the macrophages than the unoxidized particles from EtOsterilized UHMWPE. The surface reactivity of the particles also depends on the properties of the absorbed molecules, their hydrophilic/phobic character, and the release of radicals which can react with human tissues [24]. Many modifications can occur: freshly detached particles are different from particles that have been in contact with biological tissues for some time [23], and the debris may be not only fragments of UHMWPE but fragments of an oxidized, lower molecular mass polyethylene [24].

Actually, catastrophic failures due to extreme wear and heavy oxidation are quite uncommon; nevertheless, wear is also a function of time, and therefore abrasion and the production of abraded particles remains a problem in young, active patients with long life expectancies. Crosslinked UHMWPE appears to be the answer to the wear issue.

\section{Crosslinked UHMWPE}

Polymer "crosslinking" is a well-known process in chemistry: it involves the linking of two or more molecular chains through chemical covalent bonds. Amongst the several methods that can be employed to achieve this, crosslinking is obtained in orthopaedics by high-energy irradiation, which leads to the formation of radical species that react with chain imperfections and other radicals. Such reactions result in polymer chains with stable $\mathrm{C}-\mathrm{C}$ chemical bonds, theoretically increasing the molecular mass to infinity [13, 25]. Basically, crosslinked UHMWPE (XPE) has much better wear resistance and decreased mechanical properties compared to conventional UHMWPE [26].

The potential benefits of reduced particulate wear generation led to the introduction of crosslinked UHMWPE in orthopaedics during the late 1970s [27]. Following laboratory wear tests that confirmed the theoretical decrease in the wear rate, XPE has been widely used since the late 1990 s as a bearing surface for orthopaedic implants [28, 29].

Medical-grade crosslinked polyethylenes for orthopaedics are processed with radiation doses of 60-100 kGy at different temperatures and are then thermally treated to remove residual radicals. These processes vary depending on the manufacturer. The thermal treatment involves "remelting" when the temperature is above the melting point $\left(150^{\circ} \mathrm{C}\right)$ and "annealing" when below. One of the major advantages of post-irradiation thermal treatment is that it also imparts oxidation resistance to the material, due to the removal of detectable amounts of residual free radicals and hydroperoxides. Nevertheless, only melting is completely effective at eliminating the residual free radicals and the hydroperoxides formed during radiation sterilization, and therefore at preserving UHMWPE from radiation-related oxidative degradation [30, 31]. In contrast, the problem with complete melting is the resulting deterioration in mechanical properties like elongationto-break, tensile modulus, tensile strength [28, 32] and J-integral fracture toughness [32], and resistance to fatigue crack propagation [33-37].

UHMWPE melting erases the thermal history induced by ram extrusion and compression moulding; since cooling or recrystallization after melting are carried out without applying any pressure, the process decreases the overall degree of crystallinity of radiation-crosslinked UHMWPE. In theory, one possible method of restoring crystallinity in crosslinked UHMWPE would be to utilize high-pressure crystallization, but this is not possible using current processing technology.

Several new methods are now currently used in order to resolve the crystallization issue and to impart higher mechanical properties to the crosslinked polyethylene, like annealing close to but below the melting temperature of crosslinked UHMWPE [37], solid-state deformation followed by annealing [38], and repetitive subsequent annealings. All of these processes have the advantage of substantially decreasing the free radical concentration. However, detectable levels of free radicals still persist in the material, and so it still has a lower resistance to 
oxidation than remelted crosslinked UHMWPE; this is undesirable, since it would expose the material to degradative oxidation, which can also be very effective at causing dramatic insert failures for crosslinked materials.

In conclusion, crosslinked polyethylenes can have very different mechanical properties due to the different crosslinking processes that are possible, while UHMWPE, if processed and sterilized correctly, always has the mechanical property values required by the ASTM. Despite the variability, it should be noted that crosslinked UHMWPE inserts have better mechanical properties than the standard required. The mechanical properties, and the resistance to fatigue crack propagation in particular, are important in joint component applications, and so it is still unclear whether the benefits of wear resistance due to crosslinking would outweigh the risk of fatigue failure over the long term; to our knowledge, there are no reports of fatigue failures of crosslinked implants. Concerns remain about the oxidation rate of the nonremelted crosslinked inserts.

It has been demonstrated that wear particles generated by crosslinked polyethylenes play a different role in biological reactions than those generated by conventional polymers, although why this is so is not clearly understood. Crosslinking would generate a larger percentage of small particles $[39,40]$, which would lead to a higher release of tumor necrosis factor-alpha and therefore to a higher reactivity [41]. However, crosslinked and conventional polyethylenes would cause similar levels of cytokines, IL6 , IL- $1 \alpha$, IL- $1 \beta$ and TNF- $\alpha$ [42], and the degree of crosslinking-related osteolysis would be reduced compared to conventional osteolysis [43]. Regardless, radiation-crosslinked UHMWPE acetabular cups and tibial plateaus are now in clinical use, and it is still to be determined whether they will lead to a higher survivorship over the long term compared to conventional noncrosslinked UHMWPE, as expected.

Another advantage of using crosslinked polyethylene is the possibility, thanks to its resistance to abrasion, of reducing the thickness of the insert and consequently using larger femoral heads in THA, which reduce the dislocation rate and improve range of motion [44]. In contrast, fractures of the superior rim of the cup have been correlated to excessive thinness of the polyethylene [45].

Actually, assessments of the clinical behaviour of crosslinked UHMWPE depend on radiographic measurement analyses: these demonstrate an initial penetration of the femoral head into the crosslinked insert, followed by a decreased penetration after the first year compared to conventional inserts. The initial penetration observed in crosslinked inserts, in vivo and not in laboratory wear tests, has been explained as creep deformation and not as wear. The results indicate decreased wear for crosslinked
UHMWPE compared to conventional UHMWPE. To our knowledge, most studies have reported good crosslinked insert performance regardless of the manufacturer [11, 4651]; moreover, there is no evidence of large-scale failures of knee or hip implants with crosslinked components due to particle-induced osteolysis, only sporadic case reports [52] of fatigue failure $[45,53]$.

Surface cracking, abrasion, scratching and pitting have been reported on the articular surfaces of retrieved crosslinked acetabular liners, and these features have been explained as being due to the decreased ductility and fatigue resistance associated with extensive crosslinking [53]. In contrast, some studies state that the abovementioned microscopic damage to the surfaces of retrieved crosslinked inserts is a sign of load-induced plastic deformation of the surface, not an early sign of a future failure [54-57].

One final unclear issue regarding crosslinked polyethylene is its third-body wear resistance: it has been supposed that XPE, due to its micromorphology, could be less resistant to such wear than UHMWPE, which may be a problem when third bodies like bone fragments are present in revisions or microparticles of PMMA are present in cemented implants. Nevertheless, to our knowledge, there are no laboratory wear tests that report such a problem, and there are no reports of failures related to the presence of third bodies, so more studies are needed to clearly understand this issue.

\section{Future directions and stabilization against oxidation}

In the near future, we can expect radiation crosslinking processes to be optimized to improve the resistance to particulate wear without significantly decreasing mechanical properties [58]; these developments are of particular importance for knee arthroplasties, where high cyclic stresses can lead to fatigue wear mechanisms.

Despite the several thermal treatments proposed, as discussed above, oxidation can be a problem for crosslinked UHMWPE inserts, although to a degree that depends on the processing procedure $[38,49]$.

In several applications, such as food packaging and preservation, polyethylene is currently stabilized against oxidative degradation by adding a suitable biocompatible stabilizer: vitamin $\mathrm{E}$ or (better) its synthetic derivative, alpha-tocopherol $[59,60]$. Therefore, in order to combat oxidation in irradiated crosslinked UHMWPE, the use of a biocompatible and nontoxic antioxidant such as vitamin $\mathrm{E}$ has also been proposed [61]. This would lead to the double advantage of preventing the long-term oxidation associated with the presence of free radicals and preserving mechanical properties [62-65]; nevertheless, the use of any 
additive, including antioxidants, in medical-grade UHMWPE (ASTM F648) is prohibited, which has hindered the use of vitamin $\mathrm{E}$ in joint replacements for a long time.

A new standard related to the conditions required for the addition of vitamin E has been approved (ASTM Standard F 2695 2007: Standard Specification for Ultra-High Molecular Weight Polyethylene Powder Blended With Alpha-Tocopherol [Vitamin E] and Fabricated Forms for Surgical Implant Applications, ASTM International, West Conshohocken, PA, USA, see http://www.astm.org), even though the old regulation has not yet been cancelled.

Recently, given the efficacy of alpha-tocopherol in stabilizing UHMWPE against oxidative degradation, and due to its proven biocompatibility, vitamin $\mathrm{E}$ has been introduced at an experimental level by the FDA (regulation $510 \mathrm{~K})$ in order to develop orthopaedic implants that are resistant to oxidation $[60,66]$.

Many manufacturers are now developing crosslinked UHMWPE inserts containing vitamin E; however, some new concerns have arisen, in particular about the method by which the antioxidant is introduced into the polymer: it can be added before the irradiation [63, 67], during moulding or extrusion; or by diffusion after irradiation [62]. The disadvantages of these two methods are that, in the former, crosslinking is suppressed to a minor degree during irradiation, and in the latter, it is difficult to control the concentration and the distribution of the antioxidant. In both cases, the hypothesized advantage is that the vitamin $E$ protects the crosslinked polyethylene against oxidation $[68,69]$. At the moment, the elimination of post-irradiation melting in order to optimize the mechanical properties is just a fascinating hypothesis.

However, it must be noted that the use of vitamin $\mathrm{E}$ does not completely suppress oxidation during sterilization with high-energy radiation; it only retards the process. It should also be underlined that, even though the safety and biocompatibility of vitamin $\mathrm{E}$ is well known, this is still an additive with no clinical history in joint replacement components.

\section{Conclusions}

UHMWPE liners can serve well as bearing surfaces for joint replacements. In particular, if the sterilization and packaging processes are carried out correctly, the material has tribological and mechanical properties that can ensure long in vivo service as an articulation, greatly reduced wear, and particle biocompatibility, all without causing catastrophic ruptures and tissue reactions. In fact, to our knowledge, there are no reports of failures related to the mechanical properties of components made of EtO-sterilized UHMWPE and used for arthroplasties. Since the processing techniques play a fundamental role in the durability of implants, the manufacturer could be considered to be the main agent responsible implant durability.

For the same reasons, orthopaedic surgeons must pay careful attention to the processes to which the insert have been subjected: for example, $\gamma$ and electron beam irradiation can further produce oxidation, even when conducted in the absence of oxygen. The full processing history (sterilization, packaging, time of storage) of the implant, an indication of its integrity, must be present by law on the labels accompanying it, and must be considered when selecting the joint prosthesis, both before and during surgery. In fact, in cases where the surgeon is called upon to explain his choice of implant, a complete knowledge of the materials involved and the reasons for choosing the particular implant selected can be helpful.

New and promising materials, like crosslinked and vitamin E charged polyethylenes, are now considered safe but innovative and are therefore handled cautiously: many in vitro tests and several in vivo demonstrations have confirmed the validity of these materials, but it is important to remember that they do not yet have long-term clinical histories.

Open issues include: the role of debris of crosslinked polyethylene, the quantity and reactivity of which are still to be elucidated, the long-term behaviour of crosslinked material under the kinds of mechanical stresses encountered in knee arthroplasties, and the interaction of vitamin E with the surrounding tissues as well as its long-term effects on crosslinked and noncrosslinked polyethylene.

In conclusion, surgeons can use the innovative and promising products available on the market, but they should also be aware that some products have been tested over the long term and are safe for clinical use while others have short clinical histories and require caution.

Conflict of interest statement The authors declare that they have no conflict of interest related to the publication of this manuscript.

\section{References}

1. Kurtz SM, Muratoglu OK, Evans M et al (1999) Advances in the processing, sterilization, and crosslinking of ultra-high molecular weight polyethylene for total joint arthroplasty. Biomaterials 20(18):1659-1688

2. Besong AA, Tipper JL, Ingham E et al (1998) Quantitative comparison of wear debris from UHMWPE that has and has not been sterilised by gamma irradiation. J Bone Joint Surg Br 80B(2):340-344

3. McKellop H, Shen FW, Lu B et al (2000) Effect of sterilization method and other modifications on the wear resistance of acetabular cups made of ultra-high molecular weight polyethylene. A hip-simulator study. J Bone Joint Surg Am 82-A(12):17081725 
4. Harris WH (2001) Wear and periprosthetic osteolysis: the problem. Clin Orthop 393:66-70

5. Takeuchi T, Lathi VK, Khan AM et al (1995) Patellofemoral contact pressures exceed the compressive yield strength of UHMWPE in total knee arthroplasties. J Arthroplasty 10(3):363368

6. Costa L, Jacobson K, Bracco P et al (2002) Oxidation of orthopaedic UHMWPE. Biomaterials 23(7):1613-1624

7. Brach del Prever EM, Crova M, Costa L et al (1996) Unacceptable biodegradation of polyethylene in vivo. Biomaterials $17: 873-878$

8. Costa L, Luda MP, Trossarelli L et al (1998) Oxidation in orthopaedic UHMWPE sterilised by gamma-radiation and ethylene oxide. Biomaterials 19(7-9):659-668

9. Costa L, Luda MP, Trossarelli L et al (1998) In vivo UHMWPE biodegradation of retrieved prosthesis. Biomaterials 19(15): 1371-1385

10. Besong AA, Hailey JL, Ingham E et al (1997) A study of the combined effects of shelf ageing following irradiation in air and counterface roughness on the wear of UHMWPE. Biomed Mater Eng 7(1):59-65

11. Jacobs CA, Christensen CP, Greenwald AS et al (2007) Clinical performance of highly cross-linked polyethylenes in total hip arthroplasty. J Bone Joint Surg Am 89-A(12):2779-2786

12. Turell MB, Bellare A (2004) A study of the nanostructure and tensile properties of ultra-high molecular weight polyethylene. Biomaterials 25(17):3389-3398

13. Costa L, Bracco P (2004) Mechanism of crosslinking and oxidative degradation of UHMWPE. In: Kurtz SM (ed) The UHMWPE handbook: ultra-high molecular weight polyethylene in total joint replacement, vol 11. Academic, New York, pp 235250

14. Bracco P, Brach del Prever EM, Cannas M et al (2006) Oxidation behaviour in prosthetic UHMWPE components sterilised with high energy radiation in a low oxygen environment. Polymer Degrad Stab 91:2030-2038

15. Bracco P, Brunella V, Luda MP et al (2006) Oxidation behaviour in prosthetic UHMWPE components sterilised with high energy radiation in the presence of oxygen. Polymer Degrad Stab 91:3057-3064

16. Bostrom MP, Bennett AP, Rimnac CM et al (1994) The natural history of ultra high molecular weight polyethylene. Clin Orthop 309:20-28

17. Birkinshaw C, Buggy M, Daly S et al (1989) The effect of gamma radiation on the physical structure and mechanical properties of ultrahigh molecular weight polyethylene. J Appl Poly Sci 38:1967-1973

18. Blunn G, Brach del Prever EM, Costa L et al (2002) Ultra high molecular-weight polyethylene (UHMWPE) in total knee replacement: fabrication, sterilisation and wear. J Bone Joint Surg 84-B(7):946-949

19. Willert HG, Buchhorn GH (1999) The biology of the loosening of hip implants (European Instructional Course Lectures, vol 4). British Editorial Society of Bone and Joint Surgery, London, pp 58-83

20. Goodman SB (2007) Wear particles, periprosthetic osteolysis and the immune system. Biomaterials 28(34):5044-5048

21. Chiba J, Schwendeman LJ, Booth RE et al (1994) A biochemical, histologic, and immunohistologic analysis of membranes obtained from failed cemented and cementless total knee arthroplasty. Clin Orthop Relat Res 299:114-124

22. Green TR, Fisher J, Stone M et al (1998) Polyethylene particles of a "critical size" are necessary for the induction of cytokines by macrophages in vitro. Biomaterials 19(24):2297-2302

23. Fubini B (1997) Surface reactivity in the pathogenic response to particulates. Environ Health Perspect 105:1013-1020
24. Brach del Prever EM, Bistolfi A, Costa L et al (2003) The biological reaction to polyethylene wear debris can be related with oxidation of the UHMWPE cups. Chir Organi Mov 88(3):291303

25. Bracco P, Brunella V, Luda MP et al (2005) Radiation induced crosslinking of UHMWPE in the presence of co-agents: chemical and mechanical characterisation. Polymer 46(24):10648-10657

26. Pruitt LA (2005) Deformation, yielding, fracture and fatigue behaviour of conventional and highly crosslinked ultra high molecular weight polyethylene. Biomaterials 26(8):905-915

27. Oonishi H, Kadoya Y (2000) Wear of high-dose gamma-irradiated polyethylene in total hip replacements. J Orthop Sci 5(3):223-228

28. McKellop H, Shen FW, Lu B et al (1999) Development of an extremely wear-resistant ultra high molecular weight polyethylene for total hip replacements. J Orthop Res 17(2):157-167

29. Muratoglu OK, Bragdon CR, O'Connor DO et al (1999) Unified wear model for highly crosslinked ultra-high molecular weight polyethylenes (UHMWPE). Biomaterials 20(16):1463-1470

30. Wannomae KK, Christensen SD, Freiberg AA et al (2006) The effect of real-time aging on the oxidation and wear of highly crosslinked UHMWPE acetabular liners. Biomaterials 27(9): $1980-1987$

31. Harris WH (2004) Highly cross-linked, electron-beam-irradiated, melted polyethylene: some pros. Clin Orthop Relat Res 429: 63-67

32. Gomoll A, Wanich T, Bellare A (2002) J-integral fracture toughness and tearing modulus measurement of radiation crosslinked UHMWPE. J Orthop Res 20(6):1152-1156

33. Baker DA, Bellare A, Pruitt L (2003) The effects of degree of crosslinking on the fatigue crack initiation and propagation resistance of orthopedic-grade polyethylene. J Biomed Mater Res A $66 \mathrm{~A}(1): 146-154$

34. Gencur SJ, Rimnac CM, Kurtz SM (2006) Fatigue crack propagation resistance of virgin and highly crosslinked, thermally treated ultra-high molecular weight polyethylene. Biomaterials 27(8):1550-1557

35. Simis KS, Bistolfi A, Bellare A et al (2006) The combined effects of crosslinking and high crystallinity on the microstructural and mechanical properties of ultra high molecular weight polyethylene. Biomaterials 27(9):1688-1694

36. Oral E, Malhi AS, Muratoglu OK (2006) Mechanisms of decrease in fatigue crack propagation resistance in irradiated and melted UHMWPE. Biomaterials 27(6):917-925

37. Medel FJ, Pena P, Cegonino J et al (2007) Comparative fatigue behaviour and toughness of remelted and annealed highly crosslinked polyethylenes. J Biomed Mater Res B Appl Biomater 83B(2):380-390

38. Kurtz SM, Mazzucco D, Rimnac CM et al (2006) Anisotropy and oxidative resistance of highly crosslinked UHMWPE after deformation processing by solid-state ram extrusion. Biomaterials 27(1):24-34

39. Affatato S, Bersaglia G, Rocchi M et al (2005) Wear behaviour of cross-linked polyethylene assessed in vitro under severe conditions. Biomaterials 26(16):3259-3267

40. Minoda Y, Kobayashi A, Sakawa A et al (2008) Wear particle analysis of highly crosslinked polyethylene isolated from a failed total hip arthroplasty. J Biomed Mater Res B Appl Biomater 86B(2):501-505

41. Fisher J, McEwen HM, Tipper JL et al (2004) Wear, debris, and biologic activity of cross-linked polyethylene in the knee: benefits and potential concerns. Clin Orthop Relat Res 428: 114-119

42. Sethi RK, Neavyn MJ, Rubash HE et al (2003) Macrophage response to cross-linked and conventional UHMWPE. Biomaterials 24(15):2561-2573 
43. Leung SB, Egawa H, Stepniewski A et al (2007) Incidence and volume of pelvic osteolysis at early follow-up with highly crosslinked and non crosslinked polyethylene. J Arthroplasty 22(6 suppl 2):134-139

44. Bragdon CR, Greene ME, Freiberg AA et al (2007) Radiostereometric analysis comparison of wear of highly cross-linked polyethylene against 36- vs. 28 -mm femoral heads. J Arthroplasty 22(6 suppl 2):125-129

45. Tower SS, Currier JH, Currier BH et al (2007) Rim cracking of the cross-linked longevity polyethylene acetabular liner after total hip arthroplasty. J Bone Joint Surg Am 89-A:2212-2217

46. Röhrl S, Nivbrant B, Mingguo L et al (2005) In vivo wear and migration of highly cross-linked polyethylene cups a radiostereometry analysis study. J Arthroplasty 20(4):409-413

47. Digas G, Kärrholm J, Thanner J et al (2007) 5-year experience of highly cross-linked polyethylene in cemented and uncemented sockets: two randomized studies using radiostereometric analysis. Acta Orthop 78(6):746-754

48. Bragdon CR, Kwon YM, Geller JA et al (2007) Minimum 6-year follow-up of highly cross-linked polyethylene in THA. Clin Orthop Relat Res 465:122-127

49. Rohr SM, Li MG, Nilsson KG et al (2007) Very low wear of nonremelted highly cross-linked polyethylene cups: an RSA study lasting up to 6 years. Acta Orthop 78(6):739-745

50. Glyn-Jones S, Isaac S, Hauptfleisch J et al (2008) Does highly cross-linked polyethylene wear less than conventional polyethylene in total hip arthroplasty? A double blind, randomized, and controlled trial using roentgen stereophotogrammetric analysis. J Arthroplasty 23(3):337-343

51. García-Rey E, García-Cimbrelo E, Cruz-Pardos A et al (2008) New polyethylenes in total hip replacement: a prospective, comparative clinical study of two types of liner. J Bone Joint Surg $\mathrm{Br}$ 90-B(2): 149-153

52. Bradford L, Kurland R, Sankaran M et al (2004) Early failure due to osteolysis associated with contemporary highly cross-linked ultra-high molecular weight polyethylene. A case report. J Bone Joint Surg Am 86-A(5):1051-1056

53. Bradford L, Baker DA, Graham J et al (2004) Wear and surface cracking in early retrieved highly cross-linked polyethylene acetabular liners. J Bone Joint Surg Am 86-A(6):1271-1282

54. Muratoglu OK, Greenbaum ES, Bragdon CR et al (2004) Surface analysis of early retrieved acetabular polyethylene liners: a comparison of conventional and highly crosslinked polyethylenes. J Arthroplasty 19(1):68-77

55. Rieker CB, Konrad R, Schön R et al (2003) In vivo and in vitro surface changes in a highly cross-linked polyethylene. J Arthroplasty $18(7$ supp 1$): 48-54$
56. Salineros MJ, Crowninshield RD, Laurent M et al (2007) Analysis of retrieved acetabular components of three polyethylene types. Clin Orthop Relat Res 465:140-149

57. Knahr K, Pospischill M, Köttig P et al (2007) Retrieval analyses of highly cross-linked polyethylene acetabular liners four and five years after implantation. J Bone Joint Surg Br 89-B(8):10361041

58. Atienza C Jr, Maloney WJ (2008) Highly cross-linked polyethylene bearing surfaces in total hip arthroplasty. J Surg Orthop Adv 17(1):27-33

59. Mallégol J, Carlsson DJ, Deschenes L (2001) Post-irradiation reactions in vitamin $\mathrm{E}$ stabilised and unstabilised HDPE. Nucl Instrum Methods Phys Res B 85(1-4):283-293

60. Al-Malaika S (2000) Vitamin E: an effective biological antioxidant for polymer stabilisation. Polym Polym Compos 8(8):537542

61. Brach del Prever EM, Camino G, Costa L et al (1994) Impianti protesici comprendente un componente di materiale plastico. Ital Pat 1271590

62. Oral E, Wannomae KK, Hawkins N et al (2004) Alpha-tocopherol-doped irradiated UHMWPE for high fatigue resistance and low wear. Biomaterials 25(24):5515-5522

63. Tomita N, Kitakura T, Onmori N et al (1999) Prevention of fatigue cracks in ultrahigh molecular weight polyethylene joint components by the addition of vitamin E. J Biomed Mater Res 48(4):474-478

64. Oral E, Christensen SD, Malhi AS et al (2006) Wear resistance and mechanical properties of highly cross-linked, ultrahigh molecular weight polyethylene doped with vitamin E. J Arthroplasty 21(4):580-591

65. Shibata N, Tomita N (2005) The anti-oxidative properties of alpha-tocopherol in gamma-irradiated UHMWPE with respect to fatigue and oxidation resistance. Biomaterials 26(29):5755-5762

66. Wolf C, Lederer K, Pfragner R et al (2007) Biocompatibility of ultra-high molecular weight polyethylene (UHMW-PE) stabilised with alpha-tocopherol used for joint endoprostheses assessed in vitro. J Mater Sci: Mater Med 18(6):1247-1252

67. Greenbaum ES, Malhi AS, Harris WH et al (2005) Characterization of irradiated blends of alpha-tocopherol and UHMWPE. Biomaterials 26(33):6657-6663

68. Wolf C, Maninger J, Lederer K et al (2006) Stabilisation of crosslinked ultrahigh molecular weight polyethylene (UHMWPE)-acetabular components with alpha-tocopherol. J Mater Sci Mater Med 17(12):1323-1331

69. Oral E, Wannomae KK, Rowell SL et al (2007) Diffusion of vitamin-E in ultrahigh molecular weight polyethylene. Biomaterials 28(35):5225-5237 